\title{
Eficacia de los criterios de identificación del hormigón en la normativa europea (EN 206) y la reglamentación española (EHE-08)
}

\author{
Effectiveness of the Identity Testing for Concrete in European Standards \\ (EN 206) and the Spanish Regulation (EHE-O8) \\ D. Revuelta $^{(*)}$, P. Carballosa ${ }^{(*)}$, J. L. García Calvo ${ }^{(*)}$
}

\section{RESUMEN}

La normativa europea y la reglamentación española establecen una serie de criterios de conformidad y aceptación para el hormigón, así como para la identificación del producto servido. La eficacia de los primeros ha sido suficientemente estudiada mediante el análisis de las Curvas Operativas de Control, que proporcionan la probabilidad de aceptación o rechazo de hormigones conformes. Sin embargo no es posible encontrar en la bibliografía trabajos similares sobre la eficacia de los criterios de identificación, a pesar de la importancia que tienen en la toma de decisiones para el fabricante, el legislador y el consumidor. En este trabajo los autores, empleando la simulación de Monte Carlo, han investigado la eficacia de los criterios de identificación europeos y españoles trazando las curvas de probabilidad de identificación de un hormigón no conforme con lo solicitado, y de la probabilidad de rechazo de un hormigón conforme al aplicar estos criterios.

Palabras clave: Hormigón; conformidad; criterios de identificación; resistencia a la compresión; curvas operativas de control; homogeneidad de la producción.

\section{ABSTRACT}

European and Spanish regulations establish a series of conformity and acceptance criteria for concrete, as well as identity testing. The effectiveness of the first ones has been sufficiently studied by means of Operative Control Curves that provide the probability of acceptance or rejection of conforming concrete. However, it is not possible to find similar studies in the literature about the effectiveness of the identity criteria, despite their relevance in the decision making process for the manufacturer, the authorities and the consumer. In this paper the authors, using Monte Carlo simulation, have investigated the effectiveness of the European and Spanish identity criteria by obtaining curves with the probability of identifying unsolicited concrete and the probability of rejecting a conforming concrete when applying the criteria.

Keywords: Concrete; conformity; identity criteria; compressive strength; operative control curves; homogeneity of production.

(*) Instituto de Ciencias de la Construcción Eduardo Torroja (IETcc-CSIC). Madrid (España).

Persona de contacto/Corresponding author: d.revuelta@ietcc.csic.es (D. Revuelta)

Cómo citar este artículo/Citation: Revuelta, D., Carballosa, P., García-Calvo, J. L. (2015). Eficacia de los criterios de identificación del hormigón en la normativa europea (EN 206) y la reglamentación española (EHE-08). Informes de la Construcción, 67(540): e126, doi: http://dx.doi.org/10.3989/ic.15.076.

Licencia / License: Salvo indicación contraria, todos los contenidos de la edición electrónica de Informes de la Construcción se distribuyen bajo una licencia de uso y distribución Creative Commons Reconocimiento no Comercial 3.o. España (cc-by-nc). 


\section{INTRODUCCIÓN}

\subsection{Modelos estadísticos de la resistencia del hormigón, criterios de conformidad, criterios de aceptación y eficacia de los criterios (curvas operativas de calidad)}

Es bien conocido que la resistencia del hormigón, por la heterogeneidad intrínseca del material y la variabilidad del proceso de fabricación, compactación y curado, no es uniforme. Para poder abordar el diseño, cálculo, fabricación y control de calidad en las estructuras de hormigón, se asume que dicha resistencia es una variable aleatoria (1) y se construye un modelo estadístico capaz de simular adecuadamente el sistema real y las incertidumbres asociadas a la multiplicidad de causas y efectos presentes. El modelo estadístico más habitual es considerar que la resistencia a la compresión de un tipo de hormigón, producido de forma regular con los mismos materiales componentes y medios de producción, sigue una distribución de probabilidad normal o gaussiana de la forma:

$$
X \sim N\left(\mu ; \sigma^{2}\right), \mu \in \mathbb{R}, \sigma \in \mathbb{R}_{+}
$$

Puesto que los valores de los parámetros poblacionales de la distribución, la media $\mu$ y la varianza $\sigma^{2}$, sólo podrían conocerse de forma exacta determinando la resistencia a la compresión del volumen total de hormigón que forma la población, para caracterizar esta última se recurre a un modelo muestral de la forma:

$$
X_{1}, X_{2}, \ldots, X_{N}, N \in \mathbb{Z}_{+}
$$

Los resultados individuales $X_{i}(i=1,2, \ldots, N)$ corresponden al resultado de resistencia obtenido como la media de la rotura de un número determinado de probetas normalizadas, en España generalmente dos, fabricadas de muestras extraídas mediante el correspondiente procedimiento sobre una amasada o unidad de fabricación del hormigón. Se asume la hipótesis de que la muestra es aleatoria simple, es decir, los resultados individuales $X_{i}$ son estadísticamente independientes e idénticamente distribuidos.

El cumplimiento de la población con un determinado requisito es lo que se conoce como criterio de conformidad. La reglamentación y normativa europeas (2) (3), incluida la española (4), establecen que el hormigón es conforme cuando la fracción de hormigón $\theta$ de la población por debajo de un determinado valor especificado en el proyecto, conocido como resistencia característica, $f_{\mathrm{ck}}$, cumple que:

$$
\theta_{a d m} \leq 5 \%
$$

Al no poder conocer $\mu$ y $\sigma^{2}$ la comprobación efectiva del criterio es imposible, por lo que los códigos proporcionan en realidad criterios de aceptación contingentes, que en función de los datos muestrales disponibles [2], permiten calificar a la población sometida a control como aceptable o rechazable. Debido a la propia naturaleza estocástica de la resistencia del hormigón, la función de aceptación es también una variable aleatoria. Rápidamente se deduce que, en función de las probabilidades de aceptación o rechazo de poblaciones conformes o no conformes, aparecen una serie de riesgos tanto para el consumidor del hormigón (riesgo de aceptar un lote

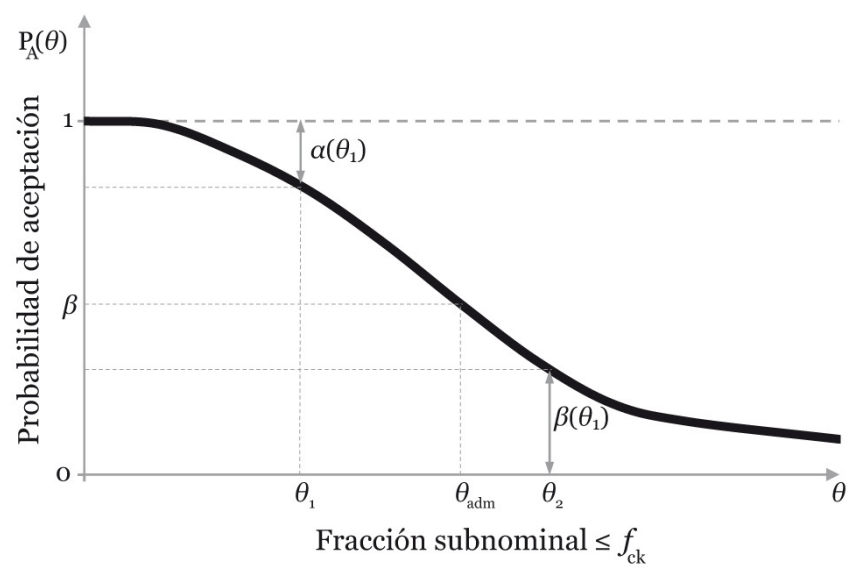

Figura 1. Curva operativa de control típica (11).

no conforme, $\beta$ ), como para el fabricante de hormigón (riesgo de que le rechacen un hormigón conforme, $\alpha$ ).

Estos conceptos fueron definidos por Dodge y Romig (5), y su conocimiento es fundamental especialmente desde el punto de vista del fabricante. Es común representar la probabilidad de aceptación del hormigón frente a la fracción subnominal por debajo del valor especificado, $f_{\text {ck }}$, para los criterios de aceptación vigentes en el lugar de suministro, en lo que se conoce como Curvas Operativas de Calidad, Curvas Operativas de Control o Curvas Características de Operación (Figura 1), según el vocabulario aceptado por la Norma UNE-ISO 3534-2 (6). Las Curvas Características de Operación indican el porcentaje de lotes que pueden ser aceptados, según los diferentes planes de muestreo, para una calidad de proceso determinada. Es decir, estas curvas indican la eficacia de los criterios de aceptación en función de la calidad del hormigón suministrado, lo que permitirá al fabricante determinar, siguiendo un criterio económico, la calidad mínima de su producción compatible con un riesgo asumible de rechazo. $\mathrm{Su}$ conocimiento es también imprescindible para el legislador, ya que será el encargado de establecer el riesgo máximo del consumidor admisible en su territorio.

Por su trascendencia económica y de seguridad, la eficacia de los criterios de aceptación o rechazo del hormigón según distintos reglamentos ha sido ya estudiada previamente en numerosas ocasiones (1) (7) (8) (9) (10) (11) (12) (13) (14), con lo que no son el objeto específico de este trabajo.

\subsection{Criterios de identificación en la normativa europea (EN 206) y la reglamentación española (EHE-o8)}

Los criterios de aceptación de la normativa europea y la reglamentación española son fundamentalmente diferentes. La comprobación de la conformidad del hormigón en España se realiza mediante control estadístico en el momento de la recepción del hormigón a la obra, recayendo la responsabilidad del control sobre la Dirección Facultativa en nombre de la propiedad. En cambio, la normativa europea EN 206 sobre conformidad del hormigón aparecida en el año 2000 (2), basa la comprobación de la conformidad en el control de producción, responsabilidad del propio fabricante. Esto abre la puerta a que la toma de muestras se pueda realizar tanto en la obra, si el transporte se considera parte del proceso de fa- 
bricación, como en la propia planta de hormigón, desligando la producción del transporte, y también se abre la posibilidad de la certificación de la producción por terceras partes, con objeto de garantizar ante los consumidores que el hormigón es aceptable conforme al criterio de la norma. No deben confundirse tampoco el control de la EN 206 con el control del distintivo de calidad oficialmente reconocido definido en la EHE-o8.

Con la filosofía planteada por la EN 206, surge entonces la siguiente pregunta: si el consumidor final del hormigón no realiza el control de aceptación, ¿cómo es capaz de reconocer que el producto que le llega es el que realmente ha solicitado y no ha habido, por ejemplo, una confusión en la transmisión de la petición? Para resolver esta inquietud, la norma europea EN 206-1 definió un concepto novedoso: los ensayos de identificación.

La filosofía de los ensayos de identificación no es la de establecer la conformidad del hormigón mediante un criterio de aceptación determinado, sino la de comprobar que el volumen de hormigón sometido al criterio de identificación pertenece a la misma población que la del hormigón aceptado como conforme mediante el control de producción establecido por el fabricante. Siguiendo este planteamiento, el riesgo del productor $\alpha$ de que un hormigón conforme sea rechazado por el criterio de identificación se establece en un valor bajo, puesto que el criterio de aceptación ya le ha sido aplicado con anterioridad. La norma cita que este riesgo $\alpha$ es del $1 \%$.

El criterio de identificación definido en la última versión de la norma EN 206 (3) establece que un hormigón se estima como proveniente de una población conforme si se cumplen simultáneamente los dos criterios de la Tabla 1. El número $N$ de resultados a comprobar se deja abierto a la decisión de los técnicos responsables de la obra.

La actual Instrucción de Hormigón Estructural española, la EHE-o8 (4), mantuvo la filosofía tradicional de control de la conformidad en la recepción, pero en un esfuerzo por converger con la normativa europea introdujo la alternativa de realizar el control de la conformidad sobre la producción. Eso sí, este control debe presentar un nivel de garantía adicional al mínimo reglamentariamente exigido por la Instrucción para el caso general, y el nivel adicional de garantía debe estar demostrado mediante la posesión de un distintivo de calidad oficialmente reconocido (D.O.R.) por una Administración.

A semejanza de la EN 206, la EHE-08 definió también un criterio de identificación, en este caso único, que se recoge en la Tabla 2. La EHE-o8 sí que establece el número mínimo $N$ de tomas a controlar.

Tal como se ha descrito previamente, existen en la bibliografía suficientes estudios como para comprender la eficacia de los criterios de aceptación definidos en la normativa europea y en la reglamentación española. Sin embargo, los autores de este trabajo no han encontrado ninguna referencia sobre el comportamiento de los criterios de identificación. Entender cómo actúan estos criterios en función de la calidad del hormigón suministrado tiene gran relevancia, tanto para el legislador como para el fabricante, por razones parecidas a las de los criterios de aceptación. Es más, desde el punto de vista del consumidor, disponer de una guía sobre la eficacia del control de identificación cobra más importancia que en el caso de los criterios de aceptación, al realizarse con una frecuencia inferior y tener una filosofía diferente (se recuerda que lo que se establece es si el suministro corresponde a lo pedido, no la calidad en sí de la producción), lo que requiere un mayor conocimiento por parte de los técnicos que han de tomar decisiones sobre qué esperar de los resultados del control de identificación.

\section{METODOLOGÍA}

\subsection{Evaluación de la eficacia de los criterios de identificación}

Ya se han descrito previamente las Curvas Operativas de Control. Para evaluar la eficacia de los criterios de identificación estudiados en este trabajo, los autores trazaron en primer lugar las Curvas Operativas correspondientes a las dos normativas, la EHE-08 y la EN 206, haciéndose la siguiente pregunta: ¿Cuál es la probabilidad de identificación de un hormigón, aplicando el correspondiente criterio, perteneciente a una población con una resistencia característica nominal $f_{\text {ck } 1}$ y una fracción subnominal $\theta$, cuando en realidad se ha solicitado un hormigón con resistencia característica

Tabla 1. Criterios de identificación para la resistencia a compresión en la EN 206 (3).

\begin{tabular}{|c|c|c|}
\hline \multirow{2}{*}{$\begin{array}{l}\text { Número } N \text { de resultados de resis- } \\
\text { tencia a compresión del volumen } \\
\text { definido de hormigón a identificar }\end{array}$} & Criterio 1 & Criterio 2 \\
\hline & $\begin{array}{l}\text { Media de } N \\
\text { resultados, } \bar{X} \\
\quad\left(N / \mathbf{m m}^{2}\right)\end{array}$ & $\begin{array}{l}\text { Cualquier resultado } \\
\text { individual, } \bar{X}_{i} \\
\left(\mathrm{~N} / \mathbf{m m}^{2}\right)\end{array}$ \\
\hline 1 & No es de aplicación & \multirow{3}{*}{$\geq f_{\mathrm{ck}}-4$} \\
\hline $2 \mathrm{a} 4$ & $\geq f_{\mathrm{ck}}+1$ & \\
\hline 5 a 6 & $\geq f_{\mathrm{ck}}+2$ & \\
\hline
\end{tabular}

Tabla 2. Criterios de identificación para la resistencia a compresión en la EHE-o8 (4).

\begin{tabular}{|c|c|c|}
\hline \multirow{2}{*}{$\begin{array}{c}\text { Resistencia característica } \\
\text { especificada en el proyecto, } \\
\boldsymbol{f}_{\text {ck }} \\
\left(N / \mathbf{m m}^{2}\right)\end{array}$} & \multirow{2}{*}{$\begin{array}{l}\text { Número } N \text { de resultados de } \\
\text { resistencia a compresión } \\
\text { del volumen definido de } \\
\text { hormigón a identificar }\end{array}$} & Criterio \\
\hline & & $\begin{array}{c}\text { Cualquier resultado } \\
\text { individual, } X_{i} \\
\left(\mathrm{~N} / \mathrm{mm}^{2}\right)\end{array}$ \\
\hline$\leq 50$ & $N \geq 1$ & \multirow{2}{*}{$\geq f_{\mathrm{ck}}$} \\
\hline$>50$ & $N \geq 2$ & \\
\hline
\end{tabular}


Tabla 3. Resistencias, criterios de identificación y número de amasadas para los casos estudiados.

\begin{tabular}{|c|c|c|c|c|c|c|c|}
\hline \multirow[b]{2}{*}{$\begin{array}{c}\text { Resistencia } \\
\text { solicitada, } \\
\boldsymbol{f}_{\text {ck2 }^{2}} \\
\left(\mathbf{N} / \mathbf{m m}^{2}\right)\end{array}$} & \multirow{2}{*}{$\begin{array}{c}\text { Resistencia } \\
\text { realmente } \\
\text { suministrada, } \\
\boldsymbol{f}_{\text {ck1 }} \\
\left(\mathrm{N} / \mathbf{m m}^{2}\right)\end{array}$} & \multicolumn{2}{|c|}{ EHE-o8 } & \multicolumn{4}{|c|}{ EN 206} \\
\hline & & Criterio & $\begin{array}{c}\text { Número de } \\
\text { amasadas } \\
\text { controladas } \\
\boldsymbol{N}\end{array}$ & Criterio & $\begin{array}{c}\text { Número de } \\
\text { amasadas } \\
\text { controladas } \\
N\end{array}$ & Criterio & $\begin{array}{c}\text { Número de } \\
\text { amasadas } \\
\text { controladas } \\
\boldsymbol{N}\end{array}$ \\
\hline 25 & 20 & \multirow{3}{*}{$X_{i} \geq f_{c k}$} & $N=1$ & $X_{i} \geq f_{c k}-4$ & $N=1$ & $\begin{array}{c}X_{i} \geq f_{c k}-4 \\
\geq f_{c k}+1\end{array}$ & $N=3$ \\
\hline 40 & 35 & & $N=1$ & $X_{i} \geq f_{c k}-4$ & $N=1$ & $\begin{array}{c}X_{i} \geq f_{c k}-4 \\
\geq f_{c k}+1\end{array}$ & $N=4$ \\
\hline 60 & 55 & & $N=2$ & $\begin{array}{c}X_{i} \geq f_{c k}-4 \\
\geq f_{c k}+1\end{array}$ & $N=2$ & $\begin{array}{c}X_{i} \geq f_{c k}-4 \\
\geq f_{c k}+2\end{array}$ & $N=6$ \\
\hline
\end{tabular}

$f_{\text {ck } 2}=f_{\text {ck } 1}+5 \mathrm{~N} / \mathrm{mm}^{2}$ ? En definitiva, se trata de determinar los riesgos del consumidor, $\beta$, pero para el caso concreto de necesidad de identificación de un hormigón perteneciente a una clase resistente un escalón inferior a la solicitada.

Las curvas se trazaron para tres niveles de resistencia característica solicitada, $f_{\text {ck2 }}$, que podrían corresponderse con un hormigón habitual en edificación, otro en obra civil, y un último hormigón de alta resistencia. Asimismo, también se estudió la posible influencia que tiene la homogeneidad de la producción en la eficacia de los criterios. Para ello, se trazaron las curvas correspondientes a poblaciones con distintos coeficientes de variación, $\delta$. El coeficiente de variación es el cociente entre la desviación estándar y la media de una población, y es un buen indicador de la calidad de las instalaciones en las que se produce el hormigón.

$$
\delta=\sigma / \mu
$$

En cuanto al número de amasadas $N$ sobre el que realizar el control de identificación, en el caso de la EHE-08 no había duda, ya que la misma Instrucción marca el número de amasadas a controlar en el caso de que el hormigón disponga de D.O.R. y le sea por tanto de aplicación el control de identificación. Puesto que la EN 206 no define el número $N$, para poder comparar la eficacia de los criterios definidos en ambas normativas se consideraron dos variantes. Por un lado, emplear el criterio de la EN 206 con el mismo número de amasadas establecidas en el control de identificación de la EHE-08 para hormigones con D.O.R., y por otro lado con el número de amasadas $N$ que establece la Instrucción de Hormigón Estructural para hormigones sin distintivo. De esta forma se podría evaluar el efecto del incremento del número de amasadas a controlar en la eficacia del criterio de la EN 206.

Las Tablas 3 y 4 contienen los valores específicos de resistencia y coeficientes de variación de la población para los que se trazaron las Curvas Operativas de Control.

Tabla 4. Coeficientes de variación analizados del hormigón suministrado.

\begin{tabular}{|c|c|}
\hline $\begin{array}{c}\text { Coeficiente } \\
\text { de variación } \boldsymbol{\delta}\end{array}$ & $\begin{array}{c}\text { Calidad } \\
\text { de la instalación }\end{array}$ \\
\hline $5 \%$ & Muy buena \\
\hline $8 \%$ & Buena \\
\hline $10 \%$ & Normal \\
\hline
\end{tabular}

Realizado el primer análisis descrito anteriormente, cabía realizarse esta otra pregunta, de incumbencia fundamental para un productor de hormigón: ¿̇cuál es la probabilidad de rechazo de un hormigón como no perteneciente a una población con la clase resistente solicitada $f_{c k}$, cuando sí que tiene esa resistencia característica nominal, en función de su fracción subnominal $\theta$ ? Este no es otro que el riesgo del productor, $\alpha$, definido previamente, y las curvas a trazar son las clásicas Curvas Operativas de Control. En esta segunda fase se estudiaron las probabilidades de rechazo para hormigones con la resistencia característica nominal igual a la solicitada en la etapa anterior $\left(25,40\right.$ y $\left.60 \mathrm{~N} / \mathrm{mm}^{2}\right)$, para los mismos coeficientes de variación y los mismos criterios y número de amasadas controladas descritos en la Tabla 3.

\subsection{La simulación de Monte Carlo}

Para poder trazar todas las curvas descritas anteriormente, los autores emplearon simulaciones de Monte Carlo (15) (16) (17) (18). El método de Monte Carlo es un procedimiento numérico que se emplea para aproximar expresiones matemáticas complejas para las que es difícil encontrar solución exacta, y que consiste en simular el comportamiento de una variable aleatoria mediante la generación de un número muy elevado de respuestas a una función aleatoria, para posteriormente computar formalmente todas las posibilidades de combinación. Es un método muy empleado en la actualidad, por el fácil acceso que se tiene a ordenadores con capacidad de generar números pseudo-aleatorios y de automatizar los cálculos de las probabilidades (19) (20).

La clave de la simulación de Monte Carlo (21) consiste en crear un modelo matemático del sistema, proceso o actividad que se quiere analizar, identificando aquellas variables (entradas del modelo) cuyo comportamiento aleatorio determina el comportamiento global del sistema. Una vez identificadas dichas entradas o variables aleatorias, se lleva a cabo un experimento consistente en generar con ayuda del ordenador muestras aleatorias (valores concretos) para dichas entradas, y posteriormente analizar el comportamiento del sistema ante los valores generados. Tras repetir $n$ veces este experimento, dispondremos de $n$ observaciones sobre el comportamiento del sistema, lo cual nos será de utilidad para entender el funcionamiento del mismo.

Para el caso estudiado, la variable aleatoria a simular es la resistencia a la compresión del hormigón, $X$, y las funciones 
son los distintos criterios en cada uno de los casos analizados (Tabla 3). A partir de [1], se deduce que (18):

$$
X=\mu+Z \sigma
$$

La variable aleatoria $X$ se generó en este caso al introducir la función ALEATORIO() de la hoja de cálculo Excel ${ }^{\mathrm{TM}}$ como argumento de la función inversa de la distribución normal estándar, INV.NORM.ESTAND().

Los datos conocidos a priori del modelo son la resistencia característica $f_{\text {ck }}$, el coeficiente de variación $\delta$, y la fracción subnominal, $\theta$, lo que nos permite calcular la media de la distribución normal de la resistencia como:

$$
\mu=\frac{f_{c k}}{1+z(\theta) \cdot \delta}
$$

y la desviación estándar $\sigma$ a partir de las ecuaciones [4] y [6].

En este trabajo se repitió el experimento un número $n=20000$ veces, se aplicaron los criterios de identificación definidos en la Tabla 3 en función de los datos de entrada, asignando el valor de $o$ en el caso de aceptación, y de 1 en caso de rechazo, y se calculó la probabilidad de rechazo (identificación).

\section{RESULTADOS Y DISCUSIÓN}

\subsection{Probabilidad de identificación de un hormigón de resistencia característica inferior a la resistencia solicitada}

La Figura 2 muestra las distintas Curvas Operativas de Control para el caso de un hormigón servido con una resistencia característica de $f_{\text {ck } 1}=20 \mathrm{~N} / \mathrm{mm}^{2}$, cuando se ha solicitado un hormigón con $f_{\text {ck2 }}=25 \mathrm{~N} / \mathrm{mm}^{2}$, para los distintos criterios seleccionados según la Tabla 3, y para distintos coeficientes de variación de la instalación de fabricación del hormigón según la Tabla 4.

La primera conclusión a la que se llega de la observación de las gráficas es que el criterio de identificación proporcionado en la EN 206, en el caso de comprobar una única amasada por volumen de hormigón a evaluar $(N=1)$, es muy poco eficaz para detectar el suministro de un hormigón de resistencia característica un nivel resistente por debajo a $\operatorname{los} 25 \mathrm{~N} / \mathrm{mm}^{2}$ solicitados, independientemente de la calidad de la instalación. Si nos fijamos en un valor de la fracción subnominal, $\theta$, del $3 \%$, valor por debajo de la fracción admisible del $5 \%$ y habitual en una planta de hormigón preparado para evitar un número antieconómico de rechazos, obtenemos que para $N=1$, la probabilidad de detectar el error en el producto solicitado es sólo del $7 \%$, 9 \% y $16 \%$, para coeficientes de variación de la producción $\delta$ del $10 \%$, $8 \%$ y $5 \%$, respectivamente.

El criterio de identificación de la EHE-o8, comprobando también una única amasada por volumen de hormigón a evaluar $(N=1)$, es mucho más eficaz para detectar un producto con $f_{\text {ck } 1}=20 \mathrm{~N} / \mathrm{mm}^{2}$ habiendo solicitado un hormigón de $f_{\text {ck2 }}=25 \mathrm{~N} / \mathrm{mm}^{2}$, ya que para $\theta=3 \%$, las probabilidades de detección del lote incorrecto son del $56 \%$, 78 \% y $100 \%$, para $\delta$ igual al $10 \%, 8 \%$ y $5 \%$, respectivamente, lo que constituyen valores razonables.
En este punto, conviene llamar la atención sobre la fuerte dependencia que tiene la eficacia de los criterios en todos los casos estudiados respecto al coeficiente de variación de la población, es decir, respecto a la homogeneidad de la producción o, lo que es lo mismo, respecto a la calidad de los medios de fabricación del hormigón, y cómo a medida que la dispersión de la población aumenta, los criterios pierden eficacia. Esta dependencia se explica muy fácilmente de forma gráfica. La Figura 3 muestra las curvas de densidad de la distribución normal de dos hormigones con la misma resistencia característica nominal, $f_{\mathrm{ck}}=20 \mathrm{~N} / \mathrm{mm}^{2}$, e idéntica fracción subnominal $\theta \operatorname{del} 3 \%$, pero con distintos coeficientes de variación. Esto quiere decir que el área de las dos curvas es la misma a la izquierda del valor de $20 \mathrm{~N} / \mathrm{mm}^{2}$. Es fácil ver cómo, para la población con mayor dispersión, la probabilidad de que algún punto quede a la derecha del valor de $25 \mathrm{~N} / \mathrm{mm}^{2}$ (criterio de identificación de la EHE-08) es muchísimo mayor que para la población con un coeficiente de variación del $5 \%$. Esta probabilidad, que no
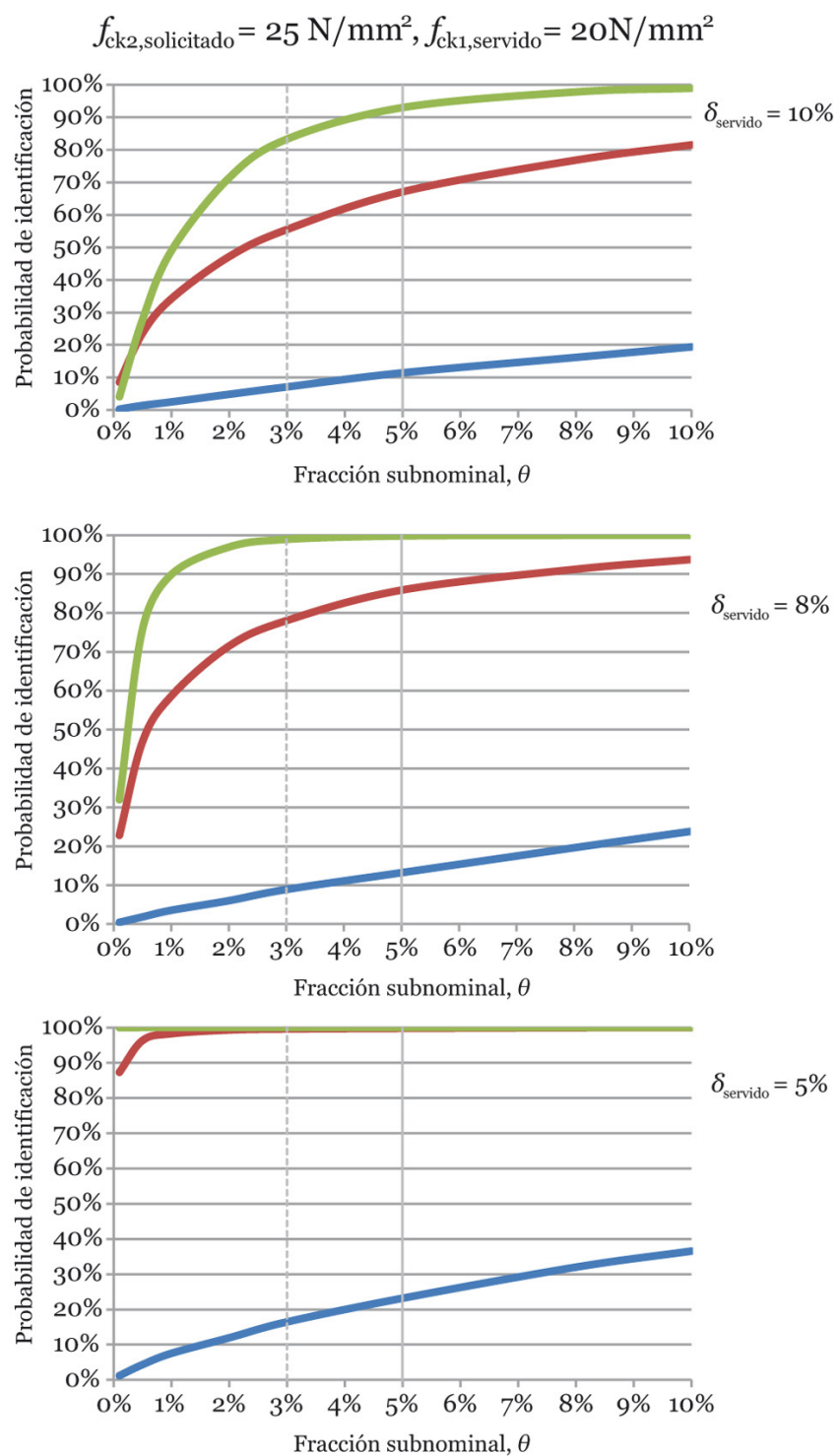

$\longrightarrow$ EHE-08 N=1 EN $206 \mathrm{~N}=1 \longrightarrow \mathrm{EN} 206 \mathrm{~N}=3$

Figura 2. Probabilidad de aceptación de un hormigón de $f_{\mathrm{ck} 1}=20 \mathrm{~N} / \mathrm{mm}^{2}$, cuando se ha solicitado un hormigón de $f_{\text {ck } 2}=25 \mathrm{~N} / \mathrm{mm}^{2}$, para distintos criterios de identificación de la Instrucción de Hormigón Estructural EHE-o8 y de la norma europea EN 206 y distintos coeficientes de variación de la producción. 


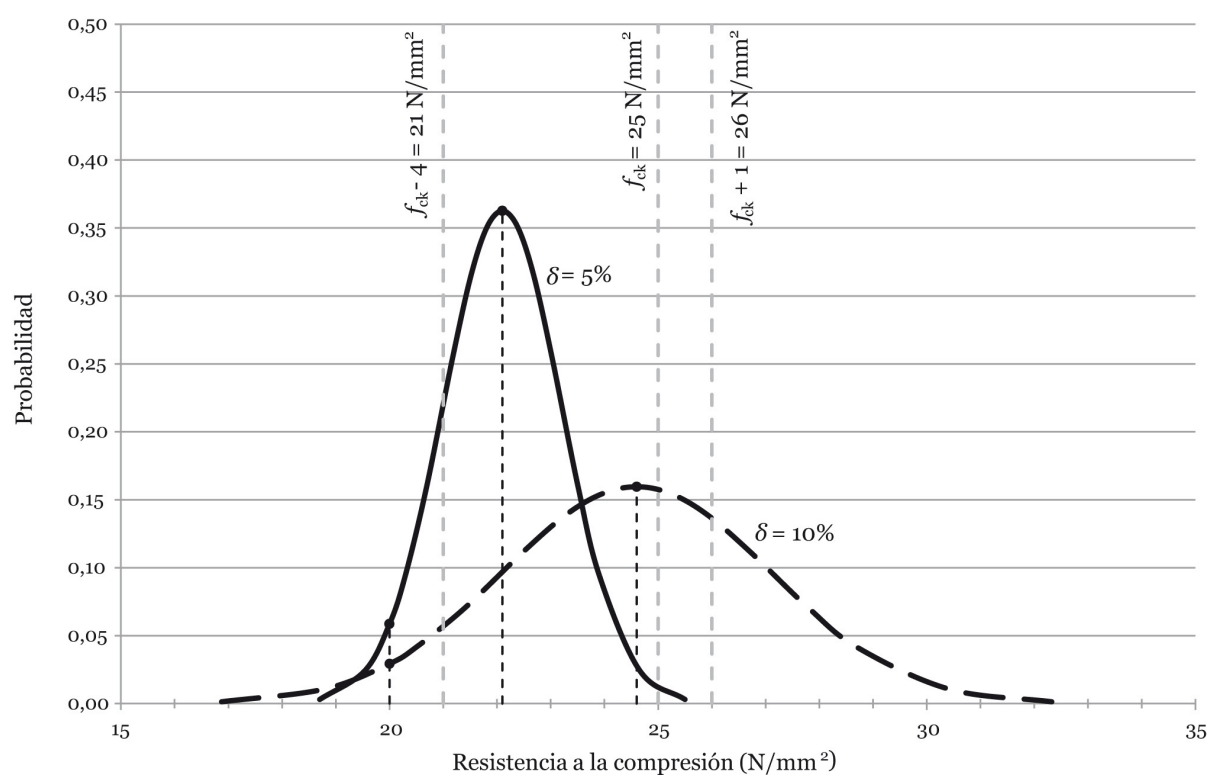

Figura 3. Curvas de distribución de densidad de dos hormigones con resistencia característica nominal $f_{\mathrm{ck}}=20 \mathrm{~N} / \mathrm{mm}^{2}$, con la misma fracción subnominal, $\theta=3 \%$, y distinto coeficiente de variación, $\delta$,

es otra cosa que el área bajo las curvas, es del 43,6 \% en el caso del hormigón con un coeficiente de variación del $10 \%$, y de tan sólo un 0,4 \% para un hormigón producido en una central con un coeficiente de variación del $5 \%$. También se comprende rápidamente por qué el criterio de identificación de la EN 206 es tan poco eficaz para el caso de ensayar una sola amasada, ya que el área a la derecha de la línea $f_{\text {ck }}-4$ es bastante considerable incluso para los hormigones con poca variabilidad.

La eficacia del criterio de identificación proporcionado por la EN 206 mejora sustancialmente al aumentar el número de tomas $(N=3)$, siendo las probabilidades de detección del $83 \%$, $99 \%$ y $100 \%$ para una fracción subnominal $\theta=3 \%$ y coeficientes de variación del $10 \%, 8 \%$ y $5 \%$ respectivamente (Figura 2). Este hecho se explica también por observación de la Figura 3. Al aumentar el número de tomas y aplicar el segundo criterio proporcionado por la $\mathrm{EN} 206$, que consiste en que la media de los resultados obtenidos sobre las amasadas controladas ha de ser superior a $f_{\mathrm{ck}}+1$, la probabilidad de detección aumenta considerablemente, ya que puede comprobarse cómo la media real está a la izquierda de ese valor para ambas distribuciones, y el estimador de la media estará centrado en torno a la media real, sobre todo a medida que aumenta el número de amasadas ensayadas. Sin embargo, es difícil que en España se emplee este último criterio proporcionado por la EN 206 para un número de amasadas superior a $N=1$, ya que en esa situación lo lógico es pensar que la Dirección Facultativa optará por el criterio de aceptación estipulado por la EHE-o8 siguiendo la modalidad del control de recepción en obra. En este trabajo se ha incluido este caso más bien como ejercicio teórico, con objeto de comprobar la eficacia de los criterios de la EN 206 a medida que aumenta el número de amasadas controladas.

Las Figuras 4 y 5 muestran las Curvas Operativas de Control de los criterios de identificación seleccionados, para hormigones solicitados de $f_{\mathrm{ck} 2}=40 \mathrm{~N} / \mathrm{mm}^{2} \mathrm{y} f_{\mathrm{ck} 2}=60 \mathrm{~N} / \mathrm{mm}^{2}$, teniendo el producto suministrado una resistencia nominal real de $f_{\text {ck } 1}=f_{\text {ck } 2}-5 \mathrm{~N} / \mathrm{mm}^{2}$. Al igual que en el caso anterior, el criterio de identificación de la EHE-o8 es más eficaz en la identificación de la población no conforme que el criterio de la EN 206 para el mismo número de amasadas controladas. También $f_{\text {ck2,solicitado }}=40 \mathrm{~N} / \mathrm{mm}^{2}, f_{\text {ck1,servido }}=35 \mathrm{~N} / \mathrm{mm}^{2}$
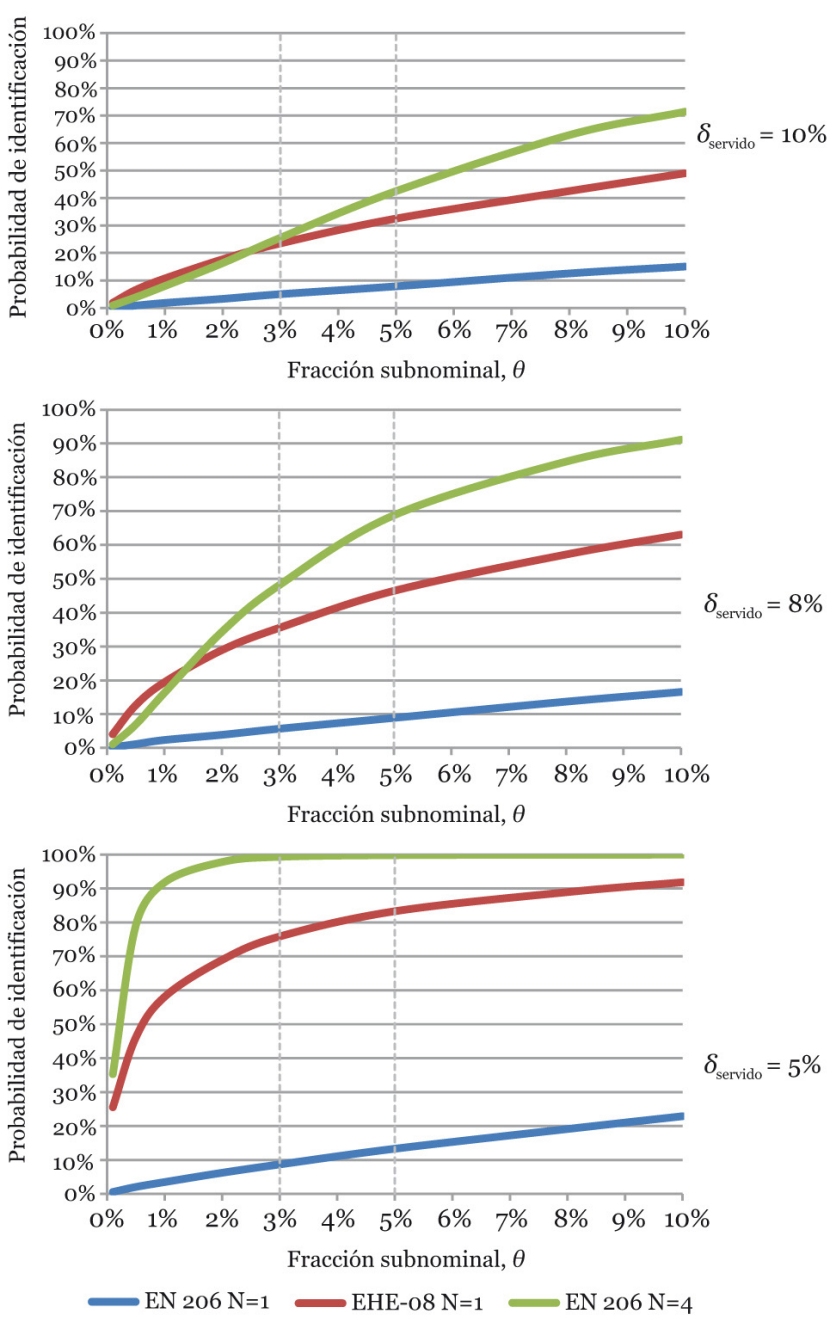

Figura 4. Probabilidad de aceptación de un hormigón de $f_{\text {ck }}=35 \mathrm{~N} / \mathrm{mm}^{2}$, cuando se ha solicitado un hormigón de $f_{\mathrm{ck}}=40 \mathrm{~N} / \mathrm{mm}^{2}$, para distintos criterios de identificación de la Instrucción de Hormigón Estructural EHE-08 y de la norma europea EN 206 y distintos coeficientes de variación de la producción. 
Tabla 5. Probabilidades de identificación de una población de hormigón con resistencia característica nominal un nivel inferior a lo solicitado $\left(f_{\mathrm{ck} 1}=f_{\mathrm{ck} 2}-5 \mathrm{~N} / \mathrm{mm}^{2}\right)$, con fracción subnominal $\theta=3 \%$, para distintos coeficientes de variación de la producción y distintos criterios.

\begin{tabular}{|c|c|c|c|c|c|c|c|c|c|c|}
\hline \multirow{3}{*}{$\begin{array}{c}\text { Resistencia } \\
\text { solicitada, } \\
f_{\text {ck2 }} \\
\left(\mathbf{N} / \mathbf{m m}^{2}\right)\end{array}$} & \multirow{3}{*}{$\begin{array}{c}\text { Resistencia } \\
\text { realmente } \\
\text { suministrada, } \\
f_{\text {ck1 }}\left(\mathrm{N} / \mathbf{m m}^{2}\right)\end{array}$} & \multicolumn{9}{|c|}{ Coeficiente de variación de la población } \\
\hline & & \multicolumn{3}{|c|}{$\delta=10 \%$} & \multicolumn{3}{|c|}{$\delta=\mathbf{8} \%$} & \multicolumn{3}{|c|}{$\delta=5 \%$} \\
\hline & & \multicolumn{9}{|c|}{ Probabilidades de identificación } \\
\hline \multirow{3}{*}{25} & \multirow{3}{*}{20} & EN 206 & EHE-o8 & EN 206 & EN 206 & EHE-08 & EN 206 & EN 206 & EHE-o8 & EN 206 \\
\hline & & $N=1$ & $N=1$ & $N=3$ & $N=1$ & $N=1$ & $N=3$ & $N=1$ & $N=1$ & $N=3$ \\
\hline & & $7 \%$ & $56 \%$ & $83 \%$ & $9 \%$ & $78 \%$ & $99 \%$ & $16 \%$ & $100 \%$ & $100 \%$ \\
\hline \multirow{3}{*}{40} & \multirow{3}{*}{35} & EN 206 & EHE-o8 & EN 206 & EN 206 & EHE-08 & EN 206 & EN 206 & EHE-o8 & EN 206 \\
\hline & & $N=1$ & $N=1$ & $N=4$ & $N=1$ & $N=1$ & $N=4$ & $N=1$ & $N=1$ & $N=4$ \\
\hline & & $5 \%$ & $23 \%$ & $26 \%$ & $6 \%$ & $35 \%$ & $48 \%$ & $9 \%$ & $76 \%$ & $99 \%$ \\
\hline \multirow{3}{*}{60} & \multirow{3}{*}{55} & EN 206 & EHE-o8 & EN 206 & EN 206 & EHE-o8 & EN 206 & EN 206 & EHE-o8 & EN 206 \\
\hline & & $N=2$ & $N=2$ & $N=6$ & $N=2$ & $N=2$ & $N=6$ & $N=2$ & $N=2$ & $N=6$ \\
\hline & & $12 \%$ & $24 \%$ & $23 \%$ & $17 \%$ & $32 \%$ & $27 \%$ & $56 \%$ & $65 \%$ & $86 \%$ \\
\hline
\end{tabular}
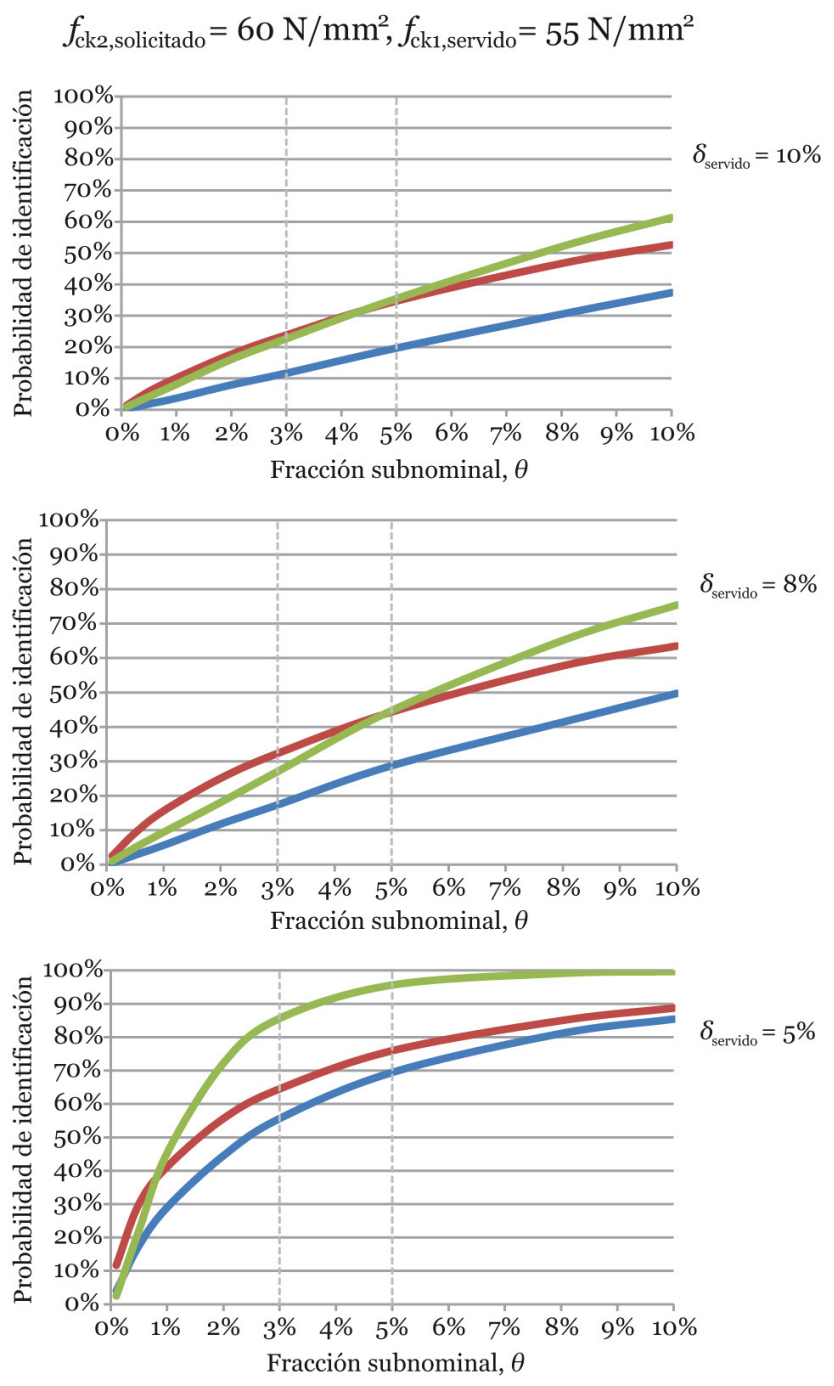

$\longrightarrow \mathrm{EN} 206 \mathrm{~N}=2 \longrightarrow \mathrm{EHE}-08 \mathrm{~N}=2 \longrightarrow \mathrm{EN} 206 \mathrm{~N}=6$

Figura 5. Probabilidad de aceptación de un hormigón de $f_{\text {ck1 }}=55 \mathrm{~N} / \mathrm{mm}^{2}$, cuando se ha solicitado un hormigón de $f_{\mathrm{ck} 2}=60 \mathrm{~N} / \mathrm{mm}^{2}$, para distintos criterios de identificación de la Instrucción de Hormigón Estructural EHE-08 y de la norma europea EN 206 y distintos coeficientes de variación de la producción. se observa de nuevo que, a medida que la homogeneidad de la población es mayor, los criterios de identificación se vuelven más eficaces, por la misma razón explicada previamente mediante la Figura 3. Resulta curioso comprobar cómo, para hormigones de alta resistencia, el criterio proporcionado por la EHE-o8 para $N=2$ tiene una eficacia similar que el criterio de la EN 206 para $N=6$, para las poblaciones habitualmente suministradas y en instalaciones de calidad normal y buena. Por último, también destaca cómo, a medida que aumenta la resistencia característica nominal, disminuye la probabilidad de detección y por tanto la eficacia de los criterios para todos los casos. Este hecho es normal puesto que, según el planteamiento del trabajo, al investigar únicamente la posibilidad de detección de hormigones con resistencia característica nominal de $f_{\text {ck }}-5 \mathrm{~N} / \mathrm{mm}^{2}$ respecto a lo verdaderamente solicitado, al aumentar la resistencia especificada disminuye en realidad la diferencia en términos relativos de la resistencia media y característica de la población suministrada respecto a lo solicitado, y por tanto se vuelve más difícil de detectar. La Tabla 5 resume las probabilidades de identificación de los diferentes casos estudiados, para una fracción subnominal de $\theta=3 \%$, habitual en producción en hormigones preparados en central.

Lo analizado hasta aquí demuestra la importancia que tiene la homogeneidad de la población, es decir, la calidad de las instalaciones de fabricación, en la eficacia de los criterios de identificación, especialmente a medida que aumenta la resistencia característica solicitada. Para profundizar en el análisis de esta influencia, la Figura 6 muestra las curvas con la probabilidad de aceptación de hormigones de resistencia característica $20 \mathrm{~N} /$ $\mathrm{mm}^{2}, 35 \mathrm{~N} / \mathrm{mm}^{2}$ y $55 \mathrm{~N} / \mathrm{mm}^{2}$, frente al coeficiente de variación $\delta$ de la población de hormigón suministrado, cuando se han solicitado hormigones de $25 \mathrm{~N} / \mathrm{mm}^{2}, 40 \mathrm{~N} / \mathrm{mm}^{2}$ y $60 \mathrm{~N} / \mathrm{mm}^{2}$, respectivamente. Todas las poblaciones poseen una fracción subnominal de $\theta=3 \%$. Se comprueba cómo el criterio de identificación proporcionado en la EN 206 es poco eficaz cuando se ensaya un número reducido de amasadas. El criterio de identificación establecido en la EHE-08, en cambio, es bastante más eficaz a igualdad de amasadas ensayadas que con el criterio de la EN 206, especialmente cuando las instalaciones de fabricación del hormigón proporcionan una homogeneidad muy buena, con coeficientes de variación en torno al $5 \%$. El empleo del criterio de la EN 206 con un número mayor de amasadas, aunque 

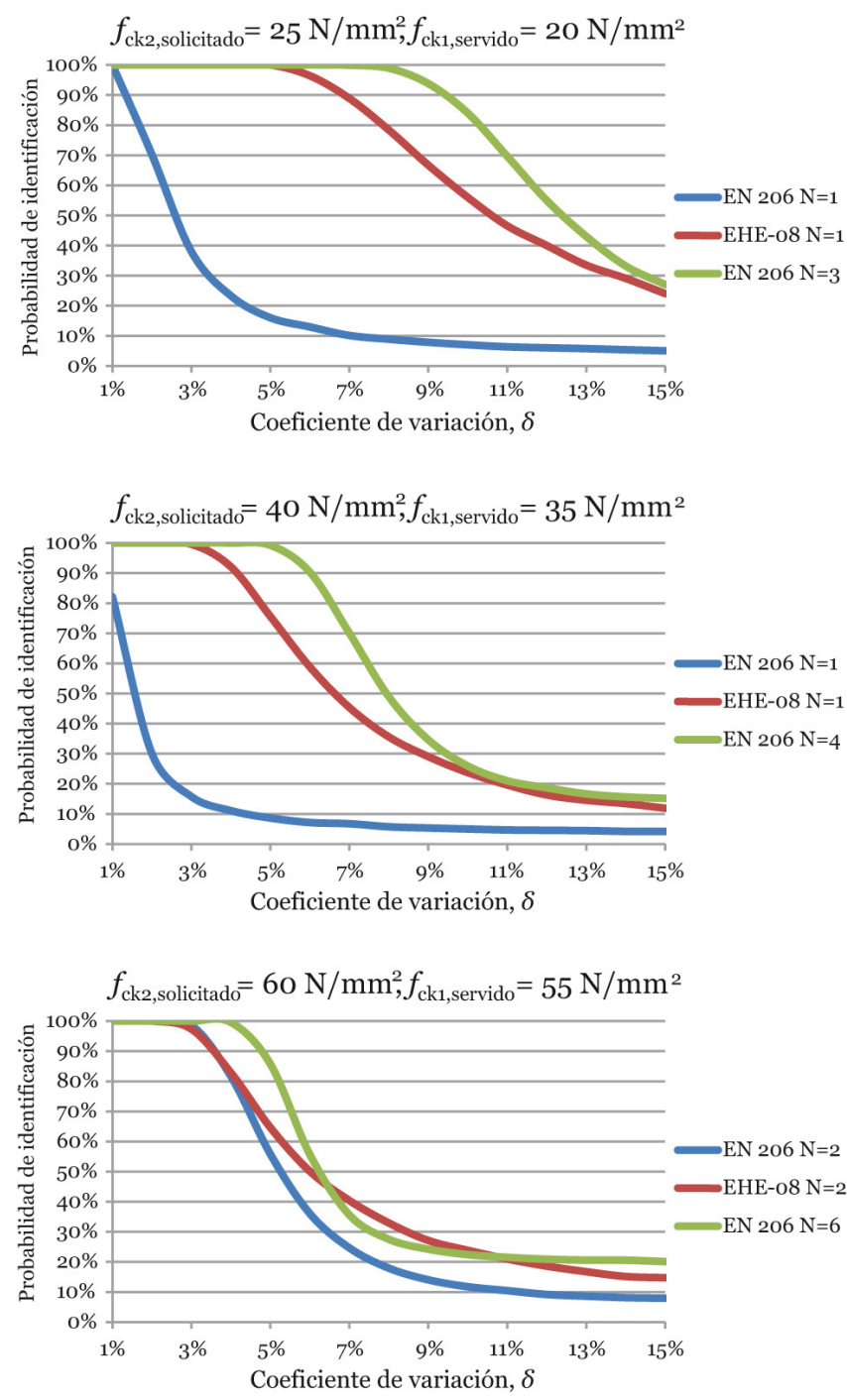

Figura 6. Probabilidad de identificación de distintas poblaciones de hormigón con resistencia característica nominal $f_{\mathrm{ck} 1}=f_{\mathrm{ck} 2}-5 \mathrm{~N} / \mathrm{mm}^{2}$ respecto a la resistencia característica solicitada, para una fracción subnominal $\theta=3 \%$ y en función del coeficiente de variación de la población.

mejora la probabilidad de identificación, no lo hace de forma especialmente significativa respecto al criterio de la EHE-o8. En todos los casos, al aumentar la resistencia solicitada la eficacia disminuye, ya que se hace más difícil detectar una diferencia menor en términos relativos respecto a la resistencia solicitada. También en todos los casos, cuanto mayor es la heterogeneidad en la fabricación, la probabilidad de detección de un volumen no perteneciente a la población solicitada disminuye. La pérdida de eficacia depende del nivel resistente del hormigón solicitado y del coeficiente de variación de la población producida. En hormigones de $f_{\text {ck }}$ solicitada hasta los $50 \mathrm{~N} / \mathrm{mm}^{2}$ es especialmente significativa a partir de coeficientes de variación del $7 \%-8 \%$. En el caso de hormigones de alta resistencia, la eficacia de los criterios está asegurada prácticamente sólo para instalaciones que aseguren una buena homogeneidad, en torno a $\delta=5 \%$.

\subsection{Probabilidad de rechazo de un hormigón conforme a lo solicitado}

La segunda parte del trabajo consistió en el análisis del riesgo del productor cuando se aplican los criterios de identificación
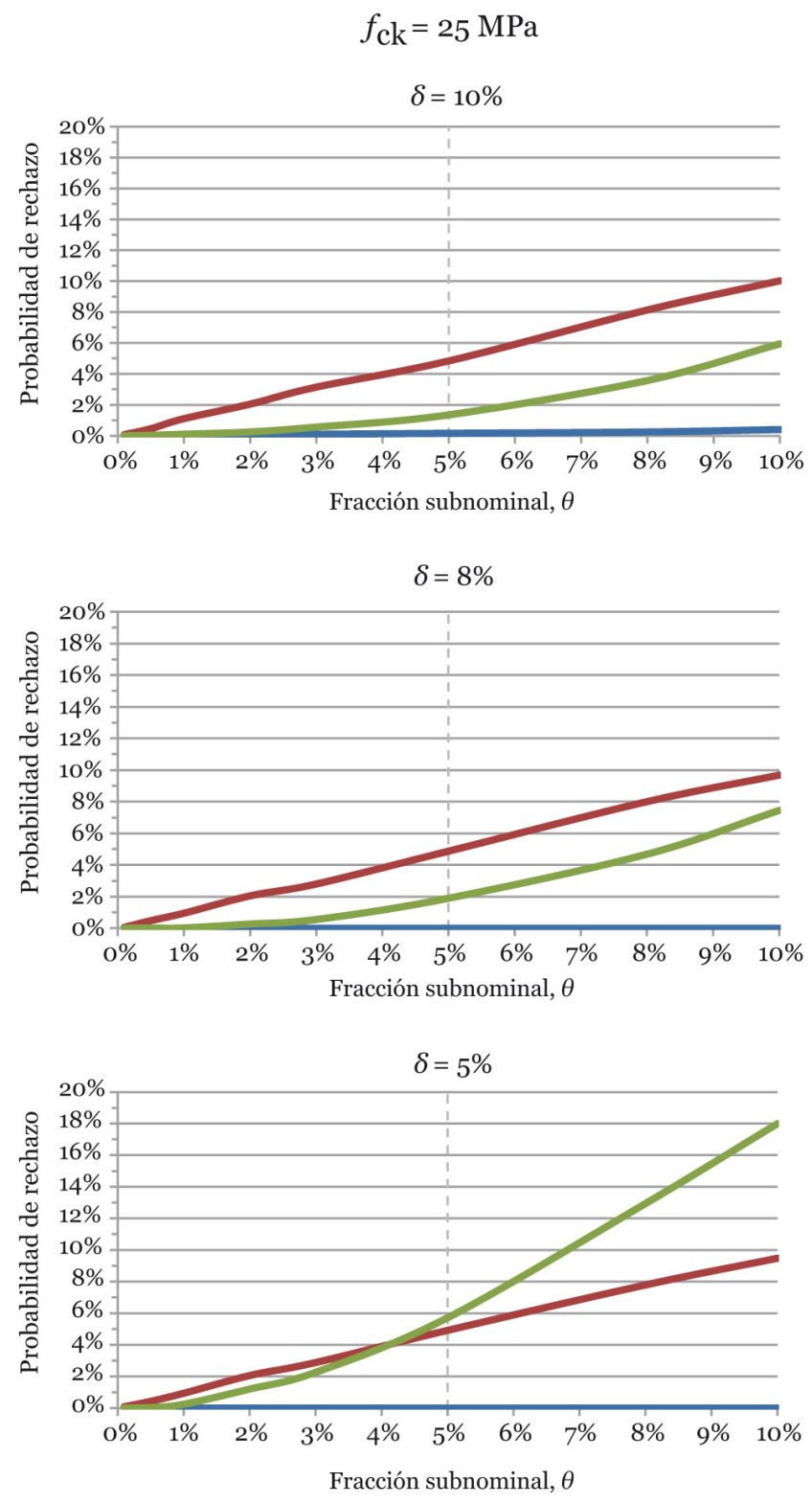

$\longrightarrow \mathrm{EN} 206 \mathrm{~N}=1 \longrightarrow \mathrm{EHE}-\mathrm{0} 8 \mathrm{~N}=1-\mathrm{EN} 206 \mathrm{~N}=3$

Figura 7. Probabilidad de rechazo de un hormigón de resistencia característica nominal $f_{\mathrm{c}}=25 \mathrm{~N} / \mathrm{mm}^{2}$, conforme a lo solicitado, para distintos criterios de identificación de la Instrucción de Hormigón Estructural EHE-08 y de la norma europea EN 206 y distintos coeficientes de variación de la producción.

de la EN 206 y la EHE-o8. La Figura 7 muestra la probabilidad de rechazo de un volumen de hormigón perteneciente a una población de resistencia característica $f_{\text {ck }}=25 \mathrm{~N} / \mathrm{mm}^{2}$, conforme a lo solicitado, para los mismos casos estudiados en el apartado anterior. La conclusión principal es que, tal como se pretende por la filosofía de los criterios de identificación, que se aplican una vez se ha sometido el hormigón a un criterio previo de aceptación con un riesgo del productor mayor, el riesgo del fabricante es apreciablemente bajo para hormigones conformes (fracción subnominal $\theta \leq 5 \%$ ), y aunque depende de $\theta$ y de $\delta$, al aplicar los criterios de identificación de la norma europea para las calidades habituales de fabricación, el riesgo del fabricante se mantiene aproximadamente en torno al $1 \%$, aunque no siempre está por debajo de ese valor tal como se declara la norma, especialmente cuando se selecciona el crite- 
Tabla 6. Probabilidades de rechazo de una población de hormigón con resistencia característica nominal igual a la solicitada, con fracción subnominal $\theta=3 \%$, para distintos coeficientes de variación de la producción y distintos criterios.

\begin{tabular}{|c|c|c|c|c|c|c|c|c|c|}
\hline \multirow{3}{*}{$\underset{\left(\mathrm{N} / \mathbf{m m}^{2}\right)}{\text { Resistencia solicitada, } \boldsymbol{f}_{\text {ck }}}$} & \multicolumn{9}{|c|}{ Coeficiente de variación de la población } \\
\hline & \multicolumn{3}{|c|}{$\delta=10 \%$} & \multicolumn{3}{|c|}{$\delta=8 \%$} & \multicolumn{3}{|c|}{$\delta=5 \%$} \\
\hline & \multicolumn{9}{|c|}{ Probabilidades de rechazo } \\
\hline \multirow{3}{*}{25} & EN 206 & EHE-o8 & EN 206 & EN 206 & EHE-08 & EN 206 & EN 206 & EHE-08 & EN 206 \\
\hline & $N=1$ & $N=1$ & $N=3$ & $N=1$ & $N=1$ & $N=3$ & $N=1$ & $N=1$ & $N=3$ \\
\hline & o \% & $3 \%$ & $1 \%$ & o \% & $3 \%$ & $1 \%$ & o \% & $3 \%$ & $2 \%$ \\
\hline \multirow{3}{*}{40} & EN 206 & EHE-o8 & EN 206 & EN 206 & EHE-08 & EN 206 & EN 206 & EHE-08 & EN 206 \\
\hline & $N=1$ & $N=1$ & $N=4$ & $N=1$ & $N=1$ & $N=4$ & $N=1$ & $N=1$ & $N=4$ \\
\hline & o \% & $3 \%$ & $1 \%$ & o \% & $3 \%$ & o \% & o \% & $3 \%$ & o \% \\
\hline \multirow{3}{*}{60} & EN 206 & EHE-o8 & EN 206 & EN 206 & EHE-08 & EN 206 & EN 206 & EHE-08 & EN 206 \\
\hline & $N=2$ & $N=2$ & $N=6$ & $N=2$ & $N=2$ & $N=6$ & $N=2$ & $N=2$ & $N=6$ \\
\hline & $2 \%$ & $6 \%$ & $5 \%$ & $2 \%$ & $6 \%$ & $3 \%$ & $1 \%$ & $6 \%$ & $1 \%$ \\
\hline
\end{tabular}

rio con múltiples amasadas, $N>1$. La probabilidad de rechazo de un hormigón conforme es algo superior cuando se aplican los criterios de identificación de la Instrucción EHE-08, pero se mantiene en valores razonablemente bajos. Así mismo, otra conclusión que se puede establecer es que la dependencia del riesgo del fabricante respecto a la homogeneidad de la población fabricada es pequeña, al menos en los rangos de fabricación conformes a los reglamentos con fracciones subnominales por debajo del $5 \%$. Esta dependencia es menor al aplicar el criterio de identificación de la EHE-o8 que el de la EN 206 de múltiples amasadas.

Estas mismas conclusiones se observaron al trazar las curvas operativas con la probabilidad de rechazo de hormigones pertenecientes a poblaciones conformes a lo solicitado con resistencias características de $40 \mathrm{~N} / \mathrm{mm}^{2}$ y $60 \mathrm{~N} / \mathrm{mm}^{2}$, resultando en curvas de forma muy similar a las de la Figura 7 , con probabilidades de rechazo mayores a medida que aumenta la resistencia característica, pero manteniéndose en valores razonablemente bajos, por lo que los autores no han estimado necesaria su inclusión en este trabajo. No obstante, la Tabla 6 presenta las probabilidades de rechazo de hormigones conformes con fracción subnominal $\theta=3 \%$, en donde se puede comprobar esta observación.

\section{CONCLUSIONES}

$\mathrm{Al}$ igual que con los criterios de aceptación establecidos en los reglamentos, la simulación de Montecarlo resulta útil para trazar las Curvas Operativas de Control de los criterios de identificación establecidos en la normativa europea y en la reglamentación española. A partir de dichas curvas, pueden establecerse las siguientes conclusiones principales:

El criterio de identificación proporcionado en la norma europea EN 206 (3) para la detección de volúmenes de hormigón no pertenecientes a la población declarada como conforme mediante el control de producción, es poco eficaz cuando se ensaya un número reducido de amasadas, ya que las probabilidades de detección de hormigones con resistencia característica nominal un escalón por debajo de la resistencia solicitada son bajas. Este criterio es aún menos eficaz al compararlo con los otros dos criterios de identificación considerados en el estudio, especialmente para hormigones de resis- tencias por debajo de los $40 \mathrm{~N} / \mathrm{mm}^{2}$, con probabilidades de detección inferiores al $16 \%$.

El criterio de identificación establecido en la Instrucción de Hormigón Estructural EHE-o8 (4) para hormigones en posesión de un Distintivo de Calidad Oficialmente Reconocido (D.O.R.) posee una eficacia razonablemente buena, y muy superior a igualdad de amasadas ensayadas que la de los criterios de la EN 206. Esta eficacia es especialmente adecuada cuando la calidad de las instalaciones de producción asegura una homogeneidad de la población con coeficientes de variación en torno al $5 \%$, ya que en estos casos la probabilidad de detección de volúmenes no pertenecientes a la característica solicitada está por encima del $65 \%$, en función de la resistencia solicitada.

El aumentar el número de amasadas a controlar aplicando los criterios de la norma EN 206, aunque mejora la probabilidad de identificación, no lo hace de forma especialmente significativa respecto al criterio de la EHE-08.

La eficacia de todos los criterios posee una fuerte dependencia respecto a la homogeneidad de la población de hormigón producida, ya que a medida que aumenta la dispersión de la población se hace más difícil el detectar un hormigón como no perteneciente a la población solicitada, de ahí la importancia que tiene la selección de centros de producción con una buena calidad de los medios de instalación. En los hormigones analizados de $f_{\text {ck }}$ solicitada hasta los $40 \mathrm{~N} / \mathrm{mm}^{2}$, la pérdida de eficacia es especialmente significativa a partir de coeficientes de variación $\delta$ del $7 \%$ - $8 \%$. En el caso estudiado de hormigones de alta resistencia, $f_{\mathrm{ck}}=60 \mathrm{~N} / \mathrm{mm}^{2}$, la eficacia de los criterios está asegurada prácticamente sólo para instalaciones que garanticen una buena homogeneidad, en torno a $\delta=5 \%$.

El riesgo del fabricante de rechazo de un hormigón con las características solicitadas al someterlo al control de identificación es apreciablemente bajo para hormigones conformes en todos los casos. En el caso de la EN 206, está en torno al $1 \%$ para hormigones de resistencia habitual, y es ligeramente mayor para hormigones de alta resistencia, $2 \%$ - $3 \%$. La probabilidad de rechazo es algo superior cuando se aplican los criterios de identificación de la Instrucción EHE-08, pero se mantiene en valores razonablemente bajos, por debajo del $6 \%$. 


\section{REFERENCIAS}

(1) López Agüí, J.C. et al. (2003). Control Estadístico del Hormigón Estructural. Monografía M-7. Madrid: Asociación Científico-Técnica del Hormigón Estructural (ACHE).

(2) CEN. (2000). EN 206-1 Concrete - Part 1: Specification, performance, production and conformity. Comité Europeo de Normalización (CEN).

(3) CEN. (2013). EN 206 Concrete - Specification, performance, production and conformity. Comité Europeo de Normalización (CEN).

(4) Ministerio de la Presidencia. (2008). Real Decreto 1247/2008, de 18 de julio, por el que se aprueba la instrucción de hormigón estructural (EHE-08). Boletín Oficial del Estado, ${ }^{\circ} 203$.

(5) Dodge, H. F., Romig, H. G. (1929). A method of sampling inspection. Bell System Technical Journal, 8(4): 613-631, doi: http://dx.doi.org/10.1002/j.1538-7305.1929.tbo1240.x.

(6) AENOR-ISO. (2013). UNE-ISO 3534-2 Estadística. Vocabulario y símbolos. Parte 2: Estadística aplicada. Asociación Española de Normalización (AENOR).

(7) Taryal, M.S. (1982). Analysis of Various Compliance Criteria for Compressive Strength of Concrete. ACI Journal Proceedings, 79(2): 147-153, doi: http://dx.doi.org/10.14359/10713.

(8) Taerwe, L. (1983). Compliance Criteria for Concrete Strength. En Thoft-Christensen, P. (Ed.), Reliability Theory and Its Application in Structural and Soil Mechanics (pp. 365-375). Dordretch: Springer Netherlands.

(9) Taerwe, L. (1987). The influence of autocorrelation on OC-lines of compliance criteria for concrete strength. Materials and Structures, 20(6): 418-427, doi: http://dx.doi.org/10.1007/BFo2472492.

(10) Taerwe, L. (2001, 13-15 de Junio). Basic aspects of quality control of concrete. En Proceedings of the 13th European Congress on Ready-Mixed Concrete (Concrete Moves). (14 páginas). Berlín. ERMCO.

(11) Prada Betancourt, D.M., Revuelta Crespo, D., Fernández Luco, L., Vega Catalán, L. (2005). Riesgos para el consumidor y productor en función de los criterios de aceptación o rechazo de hormigón preparado según distintos reglamentos (parte I). Materiales de Construcción, 55(280): 71-77, doi: http://dx.doi.org/10.3989/mc.2005.v55.i280.208.

(12) Prada Betancourt, D.M., Revuelta Crespo, D., Fernández Luco, L., Vega Catalán, L. (2006). Riesgos para el consumidor y productor en función de los criterios de aceptación o rechazo de hormigón preparado según distintos reglamentos (parte II). Materiales de Construcción, 56(281): 67-71, doi: http://dx.doi.org/10.3989/mc.2006.v56.i281.93.

(13) López Agüí, J.C. (2010). Nuevas especificaciones del hormigón y su resistencia. Bases de control. Madrid: AENOR Ediciones.

(14) Caspelee, R., Taerwe, L. (2011). Probabilistic evaluation of conformity criteria for concrete families. Materials and Structures, 44(7): 1219-1231, doi: http://dx.doi.org/10.1617/s11527-010-9694-5.

(15) Liu, J.S. (2004). Monte Carlo Strategies in Scientific Computing. Nueva York: Springer-Verlag New York. Doi: http:// dx.doi.org/10.1007/978-0-387-76371-2.

(16) Robert, C.P., Casella, G. (2004). Monte Carlo Statistical Methods. Nueva York: Springer-Verlag New York. Doi: http:// dx.doi.org/10.1007/978-1-4757-4145-2.

(17) López Agüí, J.C. (2008). Guía básica para la simulación de Monte Carlo. Madrid: AENOR Ediciones.

(18) Garrido, A., Conesa, E.M. (2009). Simulación por el método de Monte Carlo para generar criterios de aceptación en el control de calidad de productos de construcción. Informes de la Construcción, 61(515): 77-85, doi: http://dx.doi. org/10.3989/ic.09.025.

(19) Gedam, S.G, Beaudet, S.T. (2000, 24-27 de Enero). Monte Carlo simulation using Excel(R) spreadsheet for predicting reliability of a complex system. En Reliability and Maintainability Symposium, 20oo. Proceedings. Annual, (pp. 188193). Los Angeles, CA: IEEE. Doi: http://dx.doi.org/10.1109/RAMS.2000.816305.

(20) Evans, J.R. (2000). Spreadsheets as a Tool for Teaching Simulation. INFORMS Transactions on Education, 1(1):27-37, doi: http://dx.doi.org/10.1287/ited.1.1.27.

(21) Faulín, J., Juan, A.A. Simulación de Monte Carlo en Excel. Projecte e-Math. Universitat Oberta de Catalunya. http:// www.uoc.edu/in3/e-math. 